\title{
Front Matter: Volume 11059
}

, "Front Matter: Volume 11059," Proc. SPIE 11059, Multimodal Sensing: Technologies and Applications, 1105901 (16 August 2019); doi: $10.1117 / 12.2541363$

SPIE. Event: SPIE Optical Metrology, 2019, Munich, Germany 


\section{PROCEEDINGS OF SPIE}

\section{Multimodal Sensing: Technologies and Applications}

Ettore Stella

Shahriar Negahdaripour

Dariusz Ceglarek

Christian Möller

Editors

26-27 June 2019

Munich, Germany

Sponsored by

SPIE

Cooperating Organsations

ELI Beamlines (Czech Republic)

HiLASE (Czech Republic)

Laserlab Europe

European Optical Society

Published by

SPIE 
The papers in this volume were part of the technical conference cited on the cover and title page. Papers were selected and subject to review by the editors and conference program committee. Some conference presentations may not be available for publication. Additional papers and presentation recordings may be available online in the SPIE Digital Library at SPIEDigitalLibrary.org.

The papers reflect the work and thoughts of the authors and are published herein as submitted. The publisher is not responsible for the validity of the information or for any outcomes resulting from reliance thereon.

Please use the following format to cite material from these proceedings:

Author(s), "Title of Paper," in Multimodal Sensing: Technologies and Applications, edited by Ettore Stella, Shahriar Negahdaripour, Dariusz Ceglarek, Christian Möller, Proceedings of SPIE Vol. 11059 (SPIE, Bellingham, WA, 2019) Seven-digit Article CID Number.

ISSN: 0277-786X

ISSN: 1996-756X (electronic)

ISBN: 9781510627970

ISBN: 9781510627987 (electronic)

Published by

SPIE

P.O. Box 10, Bellingham, Washington 98227-0010 USA

Telephone +1 3606763290 (Pacific Time) · Fax +1 3606471445

SPIE.org

Copyright (c) 2019, Society of Photo-Optical Instrumentation Engineers.

Copying of material in this book for internal or personal use, or for the internal or personal use of specific clients, beyond the fair use provisions granted by the U.S. Copyright Law is authorized by SPIE subject to payment of copying fees. The Transactional Reporting Service base fee for this volume is $\$ 21.00$ per article (or portion thereof), which should be paid directly to the Copyright Clearance Center (CCC), 222 Rosewood Drive, Danvers, MA 01923. Payment may also be made electronically through CCC Online at copyright.com. Other copying for republication, resale, advertising or promotion, or any form of systematic or multiple reproduction of any material in this book is prohibited except with permission in writing from the publisher. The CCC fee code is $0277-$ $786 \mathrm{X} / 19 / \$ 21.00$.

Printed in the United States of America by Curran Associates, Inc., under license from SPIE.

Publication of record for individual papers is online in the SPIE Digital Library.

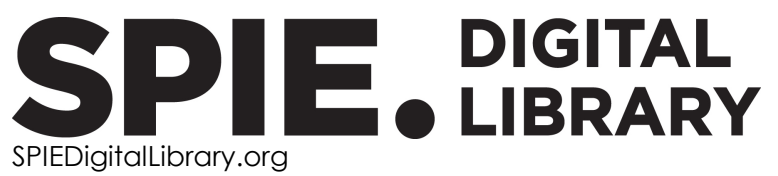

Paper Numbering: Proceedings of SPIE follow an e-First publication model. A unique citation identifier (CID) number is assigned to each article at the time of publication. Utilization of CIDs allows articles to be fully citable as soon as they are published online, and connects the same identifier to all online and print versions of the publication. SPIE uses a seven-digit CID article numbering system structured as follows:

- The first five digits correspond to the SPIE volume number.

- The last two digits indicate publication order within the volume using a Base 36 numbering system employing both numerals and letters. These two-number sets start with $00,01,02,03,04$, 05, 06, 07, 08, 09, OA, OB ... 0Z, followed by 10-1Z, 20-2Z, etc. The CID Number appears on each page of the manuscript. 


\title{
Contents
}

\author{
vii Authors \\ ix Conference Committee
}

MULTIMODAL SENSING FOR SURVEILLANCE

1105903 Radar for indoor surveillance: state of art and perspectives (Invited Paper) [1 1059-2]

1105904 Microwave imaging through an unknown wall by a MIMO configuration and SVD approach [11059-3]

1105905 Radiometer effectiveness in real cases for disclosing stealth [1 1059-4]

1105906 Passive radar for measuring passive sensors: direct signal interference suppression on FPGA using orthogonal matching pursuit and stochastic gradient descent [1 1059-5]

HOLOGRAPHY TECHNOLOGY: JOINT SESSION

1105907 Time resolved digital holography applied to droplets fragmentation by shockwave (Invited Paper) [1 1059-6]

$1105908 \quad$ How holographic imaging can improve machine learning [1 1059-7]

\section{MULTIWAVE LIGHT TECHNOLOGY}

1105909 Automated visual inspection of friction stir welds: a deep learning approach [11059-8]

11059 OA Multi-wave light technology enabling closed-loop in-process quality control for automotive battery assembly with remote laser welding (Invited Paper) [1 1059-9]

$11059 \mathrm{OB} \quad 3 \mathrm{D}$ convolutional neural networks to estimate assembly process parameters using 3D point-clouds [11059-10]

11059 OC Model-based interfacing of large-scale metrology instruments [11059-11]

$11059 \mathrm{OE} \quad$ Robust principal component analysis of ultrasonic sectorial scans for defect detection in weld inspection [1 1059-13] 
11059 OF UAV radar imaging for target detection (Invited Paper) [1 1059-14]

$11059 \mathrm{OH} \quad$ Study of complementary multi-sensors data influence on infrared thermography measurements for in situ long-term monitoring [11059-16]

11059 Ol Automatic network-level bridge monitoring by integration of InSAR and GIS catalogues [11059-17]

11059 OJ Methodology for utilization of a generalised antenna in gprMax simulator [1 1059-18]

\section{HYPERSPECTRAL IMAGING APPLICATIONS}

11059 OK Palm-sized and tough two-dimensional spectroscopic imager: the so-called hyperspectral camera for visible and mid-infrared light. Proposal of plant-species identification regardless of zenith and azimuth angles based on only two types of basic spectroscopic data (near-surface and internal reflectance) [11059-31]

$11059 \mathrm{OL} \quad$ Unsupervised feature extraction based on improved Wasserstein generative adversarial network for hyperspectral classification [1 1059-32]

11059 OM Target detection based on classification in shadow region of hyperspectral image [1 1059-33]

\section{MACHINE LEARNING APPLICATIONS}

11059 OP Multimodal data fusion for object recognition [1 1059-19]

11059 OR Challenges of designing hand recognition for a manual assembly assistance system [1 1059-21]

11059 OS Convolutional neural networks for recognition and segmentation of aluminum profiles [1 1059-22]

11059 OT Scene disparity estimation with convolutional neural networks [11059-23]

11059 OU Image acquisition, evaluation and segmentation of thermal cutting edges using a mobile device [1 1059-24]

\section{MULTIMODAL SENSING APPLICATIONS}

11059 OY An effective approach for 3D point cloud registration in railway contexts [11059-28]

$11059 \mathrm{OZ} \quad$ Multiple honey bees tracking and trajectory modeling [1 1059-29] 
1105910 New applications of electronic speckle pattern interferometry in novel materials and structures [1 1059-30]

\section{POSTER SESSION}

1105912 Study of the $360^{\circ}$ light field display [1 1059-37]

1105913 A full Stokes imaging polarimeter based on a consumer CMOS camera [1 1059-38]

1105914 Power law scaling of test error versus number of training images for deep convolutional neural networks [1 1059-39]

1105915 Research on real-time tracking algorithm of weld seam based on Fourier transform profilometry [11059-40]

1105916 Research of spatial alignment techniques for multimodal image fusion [1 1059-41]

1105917 Predictive models for abundance estimation and distribution maps of the striped dolphin Stenella coeruleoalba and the bottlenose dolphin Tursiops truncatus in the Northern Ionian Sea (North-eastern Central Mediterranean) [1 1059-42]

1105918 An electro-optical system for transverse displacement measurement with rotation parameters estimation of the measurement unit [1 1059-43]

11059 1A Floor-integrated optical fall detector for frail people [1 1059-45] 
Proc. of SPIE Vol. 11059 1105901-6 Downloaded From: https://www.spiedigitallibrary.org/conference-proceedings-of-spie on 26 Apr 2023
Terms of Use: https://www.spiedigitallibrary.org/terms-of-use 


\section{Authors}

Numbers in the index correspond to the last two digits of the seven-digit citation identifier (CID) article numbering system used in Proceedings of SPIE. The first five digits reflect the volume number. Base 36 numbering is employed for the last two digits and indicates the order of articles within the volume. Numbers start with 00, 01, 02, 03, 04, 05, 06, 07, 08, 09, OA, OB...0Z, followed by 10-1Z, 20-2Z, etc.

Adachi, Satoru, OK Akhmerov, Artyom K., 16

Amin, M., 03

Anas, Essa R., OT

Argentieri, Arturo, OS

Bachmann, A., 09

Ballesta, María, 13

Barber, T., OE

Bhattacharya, Amitabha, 0J

Bianchini Ciampoli, Luca, 이

Bianco, Vittorio, 08

Bougamale, Faysal, $\mathrm{OZ}$

Bourennane, Salah, OL, OM

Buonanno, Giovanni, 04

Calvi, Alessandro, 0 l

Carcagnì, Pierluigi, 08, 0S

Carlucci, R., 17

Cassels, B., OE

Catapano, I., OF

Ceglarek, Dariusz, $\mathrm{OA}, \mathrm{OB}$

Chatterjee, Sumona, OJ

Chrétien, Stéphane, 06

Cipriano, G., 17

Colella, Roberto, OY

D'Amico, Fabrizio, 01

dell'Aversano, Angela, 04

De Luca, Federico, OS

De Mitri, Omar, OU

Denisov, Alexander, 05

Dibari, P., 17

Dimauro, G., 17

Distante, Cosimo, 08, OS, OU

Duggal, Swati, OJ

Dumoulin, J., $\mathrm{OH}$

Esposito, G., OF

Essaïdi, Zacaria, 07

Fanizza, C., 17

Fasano, G., OF

Feng, Weike, 06

Ferraro, Pietro, 08, 10

Franciosa, Pasquale, $\mathrm{OA}, \mathrm{OB}$

Friedt, Jean-Michel, 06

Fu, Min, OM

Gabbay, Eliyahou, $0 Z$

Gagliardi, Valerio, 0 I

Gala, G., 17

Gennarelli, G., 03, OF

Gerbino, Salvatore, OA

Glorieux, Emile, OB
Goavec-Merou, Gwenhael, 06

Gorbachev, Alexey A., 18

Guo, Hualong, 12

Guo, Li, OT

Hartl, R., 09

Hartmann, Peter, 1A

Hoang, Anh Phuong, 18

Ishimaru, Ichiro, OK

Iwaki, Jyunya, OK

Jauch, Christian, OR, OU

Kang, Hanyue, OK

Kawashima, Natsumi, OK

Kitazaki, Tomoya, OK

Knyaz, Vladimir, OP

Landgraf, J., 09

Lanzotti, Antonio, OA

Lauret, Pierre, 07

Leo, Marco, OS

Li, Hongmei, 05

Liu, Hao, 05

Liu, Qingyu, 15

Liu, Xuefeng, OM

Ludeno, G., OF

Maglietta, R., 17

Magnier, Baptiste, $\mathrm{OZ}$

Maschke, Ronny, $1 \mathrm{~A}$

Matuszewski, Bogdan J., OT

Mazzeo, Pier Luigi, OS

Mein, S. J., OE

Memmolo, Pasquale, 08

Merola, Francesco, 08

Mevel, L., $\mathrm{OH}$

Mizutani, Sora, OK

Montavon, Benjamin, OC

Moradi, Behrang, $\mathrm{OZ}$

Mosca, N., 17

Nitti, Massimiliano, OY

Nixon, A., OE

Noviello, C., OF

Onsy, Ahmed, OT

Pagliarulo, Vito, 10

Patruno, Cosimo, $0 Y$

Paturzo, Melania, 08

Peña-Gutiérrez, Sara, 13

Peterek, Martin, OC

Pfister, François, $\mathrm{OZ}$

Qiu, Jinghui, 05

Rajvanshi, Tushar, 04

Renga, A., OF 
Renò, V., 17

Root, Martin, OR

Royo, Santiago, 13

Rudek, Florian, 1A

Sala, Vittorio, 14

Sato, Motoyuki, 06

Schmitt, Robert H., OC

Shark, L.-K., OE

Sinha, Sumit, OB

Slangen, Pierre R. L., 07, $0 Z$

Soldovieri, F., 03

Solimene, Raffaele, 04

Spagnolo, Paolo, OS

Spahl, J., 09

Stahl, Janek, OU

Stella, Ettore, OY

Sun, Hongwei, 15

Sun, Qiaoqiao, OL, OM

Sun, Tianzhu, OA

Taudt, Christopher, 1A

Telesca, V., 17

Tosti, Fabio, 0 I

Toullier, T., $\mathrm{OH}$

Turner, R., OE

Vasilev, Alexandr S., 16

Vasileva, Anna V., 16

Wang, Congcong, $\mathrm{OM}$

Wang, Dajing, 05

Wang, Xinghua, 15

Wu, Chao, 05

Yokoyama, Kotone, OK

Zaeh, M. F., 09

Zeng, Chong, 12

Zeng, Zhihong, 12 


\title{
Conference Committee
}

\author{
Symposium Chairs \\ Marc P. Georges, Université de Liège (Belgium) \\ Jörg Seewig, Technische Universität Kaiserslautern (Germany) \\ Honorary Symposium Chair \\ Wolfgang Osten, Universität Stuttgart (Germany) \\ Conference Chair \\ Ettore Stella, CNR (Italy) \\ Conference Co-chairs \\ Shahriar Negahdaripour, University of Miami (United States) \\ Dariusz Ceglarek, The University of Warwick (United Kingdom) \\ Christian Möller, Fraunhofer-Institut für Fertigungstechnik und \\ Angewandte Materialforschung (Germany) \\ Conference Program Committee \\ Andrei G. Anisimov, Technische Universität Delft (Netherlands) \\ Salah Bourennane, Institut Fresnel (France) \\ Cosimo Distante, Universitá del Salento (Italy) \\ Pietro Ferraro, Istituto di Scienze Applicate e Sistemi Intelligenti "Eduardo \\ Caianiello" (Italy) \\ Marc P. Georges, Université de Liège (Belgium) \\ Antonio Lanzotti, Universitá degli Studi di Napoli Federico II (Italy) \\ Luiz Marcos Garcia Gonçalves, UFRN (Brazil) \\ Michele Meo, University of Bath (United Kingdom) \\ Thomas B. Moeslund, Aalborg University (Denmark) \\ Nicola Mosca, CNR (Italy) \\ Vito Pagliarulo, Istituto di Scienze Applicate e Sistemi Intelligenti "Eduardo \\ Caianiello" (Italy) \\ Clive Roberts, The University of Birmingham (United Kingdom) \\ Pierre R. Slangen, Mines Alès (France) \\ Rocco Zito, Flinders University (Australia)
}




\section{Session Chairs}

1 Multimodal Sensing for Surveillance

Francesco Soldovieri, Istituto per il Rilevamento Elettromagnetico dell'Ambiente (Italy)

Massimiliano Nolich, Universitá degli Studi di Trieste (Italy)

JS Holography Technology: Joint Session

Pietro Ferraro, Istituto di Scienze Applicate e Sistemi Intelligenti "Eduardo Caianiello" (Italy)

Pierre R. Slangen, Mines Alès (France)

2 Multiwave Light Technology

Pasquale Franciosa, The University of Warwick (United Kingdom)

Darek J. Ceglarek, The University of Warwick (United Kingdom)

3 Multimodal Sensing for Infrastructure Monitoring

Ilaria Catapano, Istituto per il Rilevamento Elettromagnetico dell'Ambiente (Italy)

Shahriar Negahdaripour, University of Miami (United States)

4 Hyperspectral Imaging Applications

Salah Bourennane, Ecole Centrale de Marseille (France)

Nicola Mosca, Consiglio Nazionale delle Ricerche (Italy)

5 Machine Learning Applications

Cosimo Distante, Universitá del Salento (Italy)

Luiz Marcos Garcia Goncalves, UFRN (Brazil)

6 Multimodal Sensing Applications

Andrei G. Anisimov, Technische Universität Delft (Netherlands)

Michele Meo, University of Bath (United Kingdom) 